\title{
Throughput-efficient Sequential Channel Sensing and Probing in Cognitive Radio Networks Under Sensing Errors.
}

\author{
Tao Shu and Marwan Krunz \\ Department of Electrical and Computer Engineering, University of Arizona \\ Tucson, AZ 85721, USA \\ tshu@ece.arizona.edu,krunz@ece.arizona.edu
}

\begin{abstract}
In this paper, we exploit channel diversity for opportunistic spectrum access (OSA). Our approach uses channel quality as a second criterion (along with the idle/busy status of the channel) in selecting channels to use for opportunistic transmission. The difficulty of the problem comes from the fact that it is practically infeasible for a CR to first scan all channels and then pick the best among them, due to the potentially large number of channels open to OSA and the limited power/hardware capability of a CR. As a result, the CR can only sense and probe channels sequentially. To avoid collisions with other CRs, after sensing and probing a channel, the CR needs to make a decision on whether to terminate the scan and use the underlying channel or to skip it and scan the next one. The optimal use-or-skip decision strategy that maximizes the CR's average throughput is one of our primary concerns in this study. This problem is further complicated by practical considerations, such as sensing/probing overhead and sensing errors. An optimal decision strategy that addresses all the above considerations is derived by formulating the sequential sensing/probing process as a rate-of-return problem, which we solve using optimal stopping theory. We further explore the special structure of this strategy to conduct a "second-round" optimization over the operational parameters, such as the sensing and probing times. We show through simulations that significant throughput gains (e.g., about $100 \%$ ) are achieved using our joint sensing/probing scheme over the conventional one that uses sensing alone.
\end{abstract}

\section{Categories and Subject Descriptors}

C.2.1 [Computer-Communication Networks]: Network Architecture and Design-Wireless Communication

\footnotetext{
${ }^{*}$ This research was supported in part by NSF (under grants CNS-0721935, CNS-0627118, CNS-0325979, and CNS-0313234), Raytheon, and Connection One (an I/UCRC NSF/industry/ university consortium). Any opinions, findings, conclusions, or recommendations expressed in this paper are those of the author(s) and do not necessarily reflect the views of the National Science
} Foundation.

Permission to make digital or hard copies of all or part of this work for personal or classroom use is granted without fee provided that copies are not made or distributed for profit or commercial advantage and that copies bear this notice and the full citation on the first page. To copy otherwise, to republish, to post on servers or to redistribute to lists, requires prior specific permission and/or a fee.

MobiCom'09, September 20-25, 2009, Beijing, China

Copyright 2009 ACM 978-1-60558-702-8/09/09 ...\$10.00.

\section{General Terms}

Algorithms, Design, Performance, Theory

\section{Keywords}

Spectrum sensing and probing, cognitive radio networks, opportunistic spectrum access, optimal stopping theory

\section{INTRODUCTION}

\subsection{Motivation}

The benefit of opportunistic spectrum access (OSA) as a means of improving spectrum utilization is now well recognized [17]. OSA aims at opening under-utilized portions of the licensed spectrum for secondary re-use, provided that the transmissions of secondary radios do not cause harmful interference to primary radio (PR) transmissions. Because a secondary radio is supplied with more channels than what it can use for a single transmission, a critical challenge in OSA is to select in real-time the channel that the secondary radios should use. In this paper, we focus on distributed channel selection algorithms that provide a secondary radio with the maximum possible throughput under the constraint that PR transmissions are not negatively affected by this selection.

Cognitive radios (CRs) have been proposed as the enabling technology for OSA [13]. The conventional way for a CR to select channels is to scan (sense) channels and access the ones that are deemed idle. Although this approach guarantees a safe (secondary) access to the spectrum, it generally does not give optimal throughput performance. This is because the $\mathrm{CR}$ does not account for the relative quality of an idle channel, and hence it may transmit over an idle channel of poor condition, hampering the CR's throughput.

In this paper, we study a joint sensing/probing mechanism that achieves higher throughput than the classic binary channel selection approach. Under this scheme, a CR not only senses the binary status (idle/occupied) of a channel, but also probes idle channels to decide the instantaneous maximum data rates being supported on these channels. Such information is then used as a second criterion for channel selection. This mechanism is motivated by the rich channel diversity in CR environments, where signal fluctuations over various channels become independent once the channel bandwidth is greater than the signal's coherence bandwidth. For example, in an IEEE 802.22 WRAN (the first standard for CR networks ( $\mathrm{CRNs})$ ), each channel occupies a $6-\mathrm{MHz}$ bandwidth, while the signal's delay spread typically ranges between $100 \mathrm{~ns}$ and $10 \mu \mathrm{s}$ [14], corresponding to a coherence bandwidth ranging from $16 \mathrm{KHz}$ to $1.6 \mathrm{MHz}$ (coherence bandwidth $=1 /(2 \pi \times$ delay spread) $[14])$. Thus, it is possible for a $\mathrm{CR}$ to exploit this multi-channel diversity by opportunistically selecting a good channel for transmission. 


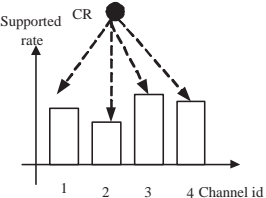

(a) Parallel channel

scan.

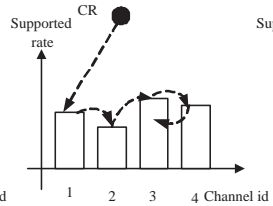

(b) Sequential channel scan with recalled channel

lection: ch3 is skipped in the

first place and then picked after scanning ch4

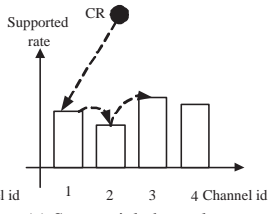
without channel recall: ch3 is selected in the first place and channel scan process terminates after that (c) Sequential channel scan

Figure 1: Various channel scan and selection paradigms.

This work focuses on the operational aspects of the above mechanism. This is in contrast to related works that study the conceptual aspects of multi-channel diversity from a highlevel mathematical standpoint and tend to ignore its operational details. Specifically, we account for the following practical considerations in developing our mechanism. First, the instantaneous condition of a channel is unknown to a CR until it is sensed and probed by that CR. Due to the potentially large number of channels and the CR's power/hardware limitations, it is infeasible for the CR to first scan all channels simultaneously and then pick the best among them. A CR's channel sensing and probing can only be conducted sequentially. After sensing and probing a channel, the CR needs to decide whether to terminate the scan and use the last scanned channel, or to skip it and scan the next one. To avoid collisions with PRs and other CRs, a CR cannot recall (use) a channel it previously skipped, because of the staleness of that sensing/probing outcome (e.g., the channel may have been occupied by other $\mathrm{CR}$ or PR, or its quality has changed). To better understand the above process, we illustrate various channel scan/selection paradigms in Figure 1, among which sub-figure (c), i.e., sequential channel scan and non-recalled channel selection, is the one we pursue in this work. Under this paradigm, the optimal use-or-skip decision strategy that maximizes the CR's average throughput is one of the key issues investigated in this paper.

The above decision making process is further complicated when the CR's sensing and probing overheads need to be accounted for in each step. Empirical data shows that sensing a channel takes tens of $\mathrm{ms}$ and probing a new one takes from 10 to $133 \mathrm{~ms}$, depending on the association and capture speed between the transmitter and receiver after each channel hopping [1]. At the same time, to reduce collisions with newly activated PRs, a CR's continuous transmission over an idle channel must be limited, e.g., at most few seconds. After that, the CR needs to sense/probe channels again. As such, the accumulated overhead after sequentially sensing/probing several channels could be comparable with or even greater than the CR's actual transmission time. When such overhead is accounted for, the gain that may be potentially achieved by looking for a slightly better channel than the currently scanned one may not be justifiable.

Furthermore, we need to account for the impact of sensing errors on the CRN throughput. Sensing errors exist in all real systems and, as shown shortly, they significantly impact the throughput. When sensing errors are present, a CR may, for example, falsely identify an idle channel as being occupied, thus missing a transmission opportunity. Under this setup, the CR's sensing time (i.e., the amount of time the CR spends on sensing a channel) becomes a variable to be optimized. Specifically, the sensing time determines the accuracy of the channel sensing process. A shorter sensing time reduces the scanning time of a channel, but also increases the probability of a false alarm. This in turn increases the number of channels the CR needs to sense and probe, leading to possibly longer overall channel search time, and thus a re- duction in throughput. As such, the tradeoff between sensing time and sensing accuracy needs to be carefully evaluated.

\subsection{Main Contributions}

We provide an integrated framework that addresses all the above practical considerations. Our main contributions are as follows. First, we derive the throughput-optimal decision strategy for the sequential channel sensing/probing process. It turns out that this optimal strategy has a threshold structure, which basically indicates whether the channel is good or bad. To properly set this threshold, we consider the tradeoff between the achievable data rate brought by good channels and the time cost (and consequently, throughput reduction) for searching for good channels. Second, we derive the maximum acceptable channel probing time that guarantees a positive throughput gain for the proposed method over a scheme that does not utilize probing. This knowledge is important because the accumulated probing time may be so significant that it cancels out gains achieved by selecting good channels. Third, we optimize the channel sensing time. It turns out that this optimization is non-convex when probing is used (and thus leads to a non-binary multi-rate setup) in the presence of sensing error. However, by exploiting the special structure of the problem, we still achieve a good solution that gives provably near-optimal performance. Our work is the first to incorporate the relationship between sensing time and sensing accuracy in an operational CR environment.

The above contributions are made by performing two rounds of optimization. In the first round, we treat the sensing and probing times as parameters, and derive the parametrically optimal probing strategy. This is achieved by formulating the sensing/probing/access process as an infinite-horizon maximum rate-of-return problem in the optimal stopping theory framework [6], with the number of bits that the CR is able to send in one transmission as the return, and the overall channel search plus transmission times as the time cost. Next, we look into the particular structure of this optimal strategy and perform a second round of optimization over the operational parameters, such as the sensing and probing times, aiming at maximizing the outcome of the first-round optimization.

Besides the above optimization aspects, we are also interested in evaluating the aggregate throughput performance when a network of CRs coexist with PRs, and each CR reacts according to its sensing/probing/access scheme in a distributed way. A Markov-chain model is developed for our performance analysis, whereby the contention between CRs, the sensing strategies employed (random channel sensing and collaborative channel sensing), and probing threshold settings at individual CRs are all accounted for. Our results show that when the sensing/probing parameters are properly set, the addition of probing can significantly improve the CRN's throughput, e.g., over $100 \%$ gains are observed in our simulations.

The remainder of this paper is organized as follows. We review related works in Section 2. Section 3 describes the system model and its maximum rate of return formulation. Using optimal stopping theory, we solve the optimization problem in Section 4. Section 5 studies the performance for the multi-CR case. Simulation results are presented in Section 6, and Section 7 concludes the paper.

\section{RELATED WORK}

Reference [2] is probably the most relevant paper to our work. In [2], the authors addressed the optimal channel selection problem under the implicit assumption that channel sensing is always accurate. As a result, the impact of sensing time on the optimization is ignored. This simplification makes the optimization relatively straightforward, because, as we explained in the previous section, the relationship be- 
tween sensing time and throughput is non-linear and nonconvex. In addition, the optimization in [2] is formulated under a utility-function objective, which characterizes the benefit of transmitting over a good channel and the overhead of finding such a channel through an additive revenuecost relationship. In contrast, our optimization is formulated as a rate of return problem, which describes the multiplicative relationship between the number of bits sent in a single transmission and the total time spent on preparing for and executing this transmission. Our formulation eliminates the ambiguity of setting the per-unit values for revenue and cost for the utility function in [2], and hence has a more meaningful physical interpretation (i.e., the average throughput achieved per transmission). The more realistic considerations pursued in this paper make our problem much harder than in [2]. It requires us not only to derive the optimal sensing/probing strategy under the new objective function, but also to look into the structure of this strategy to optimize various operational parameters.

Aside from [2], other works on exploiting channel-quality information in CRNs tend to study the problem from a conceptual standpoint, ignoring various operational details. Such simplifications may largely overestimate the benefit brought by multi-channel diversity. For example, the work in [8] suggests to maximize CRN's spectrum efficiency by adjusting CRs' sensing periods according to their channel conditions. However, it ignores various practical considerations, i.e., sequential sensing/probing, overhead, and sensing errors. It also assumes that the CR can scan all channels simultaneously. The authors in [18][5] studied OSA for a slotted system. They assumed that a CR can only sense, probe, and access one channel in one slot. If the channel is occupied or its quality is poor such that the channel is not used by the $\mathrm{CR}$, the CR is not allowed to sense/probe other channels, even though there is still big chunk of time remaining in the underlying slot. Thus, the sequential exploration of channel diversity is ignored in these works. Similarly, the work in [10] studied the optimal sensing and transmission times that maximize the so-called spectrum efficiency under an interference constraint for a single-channel system. However, their optimization has ignored the effect of a multi-channel setup, whereby the CR may scan multiple channels before starting a transmission. The works in [12] and [3] suggest a candidateset-based scheme, where each CR may precompute a subset of channels of relatively good quality in a statistical sense. When a CR needs to transmit, it only senses and picks channels from this subset. This scheme ignores the fact that in a multi-CR operational environment, the subsets calculated by various CRs may overlap significantly with each other, because similar channel conditions are observed by close-by CRs. As a result, this mechanism may lead to poor performance in a multi-CR environment due to high collisions in CRs' channel selection.

For wireless systems with dedicated channels, there have been several works that consider joint optimization of the reward obtained from channel selection and the cost incurred by channel probing (e.g., $[15,20,19,9,7,21])$. The differences between our problem and these previous works include the following: First, the problem of combined channel sensing and probing has not been considered in all these works. The inclusion of sensing in the channel selection problem is non-trivial, because the sensing time can affect the throughput by nonlinearly changing the channel sensing accuracy. Second, previous work on channel probing involves only a relatively small channel pool, e.g., a pool of 3 channels for 802.11 and 8 channels for 802.11a. For CRNs, this pool can be much larger, e.g., over 100 channels in a 802.22 WRAN. As a result, algorithms designed for small channel pools, e.g., the finite-horizon stopping method in [15] and the tree-based searching algorithm in $[7,9]$, become practically infeasible in

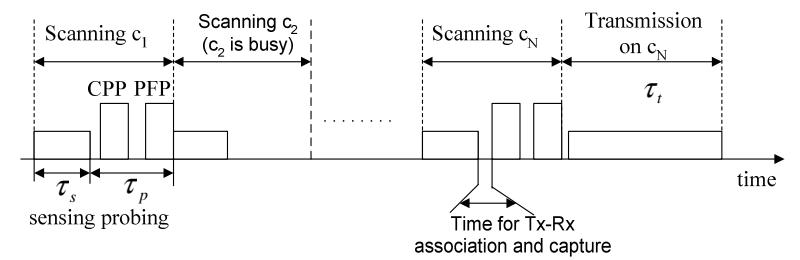

Figure 2: Sequential channel sensing and probing before transmission.

CRNs because of their prohibitive computational complexity when the number of channels is large. In our work, an infinite-horizon formulation is employed, which is particularly suitable for modeling large channel pools. Third, the ultimate concern of all previous works is the optimal probing strategy that maximizes the throughput. In this work, we are not only interested in the optimal probing strategy, but also in the particular structure of this strategy, with the objective of performing a second-round optimization over operational parameters such as the sensing and probing times.

Finally, we note that the impact of sensing accuracy on throughput has been studied in [11][4] under binary use of channels. It was shown that under this binary setup, throughput is a concave function of sensing time. In this paper, we study the problem under a more complicated multi-rate environment. We show that the problem loses its concavity in this case. One key contribution of our paper is in studying the special structure of the new problem and suggesting an approximate solution that provides provably near-optimal performance.

\section{MODEL DESCRIPTION AND PROBLEM FORMULATION}

\subsection{System Model}

We consider a set of $C$ licensed channels. The status of a channel is modeled as a continuous-time random process that alternates between two states: idle and busy. A busy (idle) state indicates that some (no) PR user is transmitting over the channel. Denote the average idle and busy durations by $\alpha$ and $\beta$, respectively. When the channel is observed at an arbitrary time, its idle and busy probabilities are given by $P_{I}=\frac{\alpha}{\alpha+\beta}$ and $P_{B}=\frac{\beta}{\alpha+\beta}$, respectively. Here we focus on the homogeneous channel utilization scenario, i.e., we assume that the states of different channels are driven by homogeneous and independent random processes. This may correspond to the scenario that all channels belong to the same licensed network. The channel selection problem under heterogeneous channel utilization is actually trivial, because in that case a CR should select the channel with the lowest utilization.

Along with the PR users, the spectrum is opportunistically available to a set of CRs. To simplify the exposure, we ignore for the time being the CR-to-CR contention issue. This allows us to focus on the channel sensing/probing/access process for a pair of CRs, a transmitter and a receiver, with the goal of optimizing this process. We also assume that some synchronization mechanism (e.g., based on random-number generaters) is in place so that the CR transmitter and receiver are always sensing and probing the same channel at the same time. We will account for the contention issue in Section 5 when we study the multi-CR scenario.

When a CR wants to transmit, it starts scanning the channels sequentially, as shown in Figure 2. Specifically, it randomly picks a channel, say $c_{1}, 1 \leq c_{1} \leq C$, and samples it for $\tau_{s}$ time. Then the CR decides whether channel $c_{1}$ is idle or busy. If it is busy, the CR randomly selects the next 
channel $c_{2}, 1 \leq c_{2} \leq C$, to sample, and so on. Suppose that in the $n$th step, channel $c_{n}$ is determined to be idle. Then the CR transmitter begins to probe that channel by sending a channel probing packet (CPP) over channel $c_{n}$ using a predefined transmission power. The $\mathrm{CR}$ receiver measures the strength of the CPP signal and decides the maximum achievable data rate (MADR), $r_{n}$, that can be supported by the current channel. The value of $r_{n}$ is selected from a set of discrete rates: $\left\{R_{k}, k=0,1, \ldots, K\right\}$, where $R_{k}$ increases with $k$ and $R_{0} \stackrel{\text { def }}{=} 0$. This MADR value is then embedded into a probing feedback packet (PFP), which is sent back from the receiver to the transmitter over channel $c_{n}$. The time spent on one $\mathrm{CPP} / \mathrm{PFP}$ exchange plus the preceding time for association and capture between the transmitter and receiver is called the channel probing time $\tau_{p}$. After receiving the PFP, the transmitter decides whether or not to use this channel. This is done by comparing $r_{n}$ with some channel quality threshold, $r^{*}$. If $r_{n} \geq r^{*}$, then the transmitter terminates the channel search and transmits at rate $r_{n}$ over channel $c_{n}$ for $\tau_{t}$ duration of time ( $\tau_{t}$ should not be too long to reduce the likelihood of collisions with newly activated PRs). If $r_{n}<r^{*}$, the CR skips this channel and continues to sense the next one. Because the CR receiver also has knowledge of $r^{*}$ (e.g., this information can be embedded into the CPP), there is no need for the transmitter to notify the receiver about its decision. Note that if the channel is busy during the sensing phase, no probing packets should be exchanged between the CR transmitter and receiver, to avoid interfering with PRs. However, the receiver still has to wait for $\tau_{p}$ time to realize that the channel is busy ${ }^{1}$. Therefore, whether or not the channel is idle, the time cost for one step of channel search is $\tau_{s}+\tau_{p}$.

Remark: If a CR can transmit over $J$ idle channels at a time, $1 \leq J \ll C$, then $J$ parallel channel scanning/access instances can be initiated and maintained by the CR. Each instance will independently search and use one idle channel according to the above sequential process. Therefore, we only need to focus on one such instance in our treatment.

Channel sensing is modeled as a binary hypothesis test, where $H_{0}$ indicates an idle channel and $H_{1}$ indicates an occupied channel. Let $x(t)$ be the sample collected by the CR. Then,

$$
x(t)= \begin{cases}n(t), & H_{0} \text { (idle) } \\ s(t)+n(t), & H_{1} \text { (occupied) }\end{cases}
$$

where $n(t)$ is the AWGN and $s(t)$ is the received PR's signal at the CR. For this sensing process, the probabilities of false alarm $P_{f a}$ and miss detection $P_{m d}$ are defined as follows:

$$
\begin{aligned}
& P_{f a}\left(\tau_{s}\right)=\operatorname{Pr}\left\{\mathrm{CR} \text { decides the channel is busy } \mid H_{0}\right\} \\
& P_{m d}\left(\tau_{s}\right)=\operatorname{Pr}\left\{\mathrm{CR} \text { decides the channel is idle } \mid H_{1}\right\} .
\end{aligned}
$$

Note that these two probabilities, which represent sensing accuracy, are functions of the sensing time $\tau_{s}$.

The unconditional probabilities that a CR determines that the channel is idle $\left(Q_{I}\right)$ or busy $\left(Q_{B}\right)$ are given by:

$$
\begin{gathered}
Q_{I}=P_{B} P_{m d}+P_{I}\left(1-P_{f a}\right) \approx P_{I}\left(1-P_{f a}\right) \\
Q_{B}=P_{B}\left(1-P_{m d}\right)+P_{I} P_{f a} \approx P_{B}+P_{I} P_{f a}
\end{gathered}
$$

The approximation in the above equations is due to the practical requirement that $P_{m d} \ll 1$ (e.g., $1 \%$ is a typical value), which ensures negligible interference from CRs.

\footnotetext{
${ }^{1}$ Depending on the distance between the transmitter and the receiver, they may sense different status for a channel. The fixed $\tau_{s}+\tau_{p}$ scanning time guarantees perfect tx-rx synchronization under any case.
}

\subsection{Problem Formulation}

The throughput-optimal sequential channel sensing/probing/ access process can be formulated as an optimal stopping problem. We first briefly describe the definition of an optimal stopping problem and then present our formulation.

An optimal stopping problem is defined by the following two components [6]:

1. A sequence of random variables (rvs) $X_{1}, X_{2}, \ldots$, whose joint distribution is assumed to be known.

2. A sequence of real-valued reward functions, $y_{0}, y_{1}\left(x_{1}\right)$, $y_{2}\left(x_{1}, x_{2}\right), \ldots, y_{\infty}\left(x_{1}, x_{2}, \ldots\right)$.

The rvs $X_{1}, X_{2}, \ldots$, can be observed sequentially (one variable at a time) for as long as needed. For each observation instance $n=1,2, \ldots$, after observing $X_{1}=x_{1}, X_{2}=$ $x_{2}, \ldots, X_{n}=x_{n}$, one may stop and receive the known reward $y_{n}\left(x_{1}, \ldots, x_{n}\right)$, or one may continue to observe $X_{n+1}$. If no observations are made, the received reward is the constant $y_{0}$. If the observer never stops, the received reward is $y_{\infty}\left(x_{1}, x_{2}, \ldots\right)$. The goal is to choose a rule to stop such that the expected reward at the stopping time $N, E\left\{y_{N}\right\}$, is maximized. According to this framework, the optimal-stopping formulation of our problem is as follows.

First, we define the sequence of observations. For the $n$th sensing and probing step, $n \geq 1$, the MADR value of the channel, $r_{n} \in\left\{0, R_{1}, \ldots, R_{K}\right\}$, can be obtained. Let the distribution of $r_{n}$ be $p_{k}=\operatorname{Pr}\left\{r_{n}=R_{k}\right\}, k=0,1, \ldots, K$ (we assume the fluctuations on different channels are i.i.d.). This distribution depends on fading and shadowing effects on the channel. We define $X_{n}$ as the outcome of the $n$th sensing and probing step: $X_{n}=0$ if the channel is busy and $X_{n}=r_{n}$ if the channel is idle. The distribution of $X_{n}$ can be calculated as follows.

$$
\begin{gathered}
q_{0} \stackrel{\text { def }}{=} \operatorname{Pr}\left\{X_{n}=0\right\}=Q_{B}+Q_{I} p_{0} \\
q_{k} \stackrel{\text { def }}{=} \operatorname{Pr}\left\{X_{n}=R_{k}\right\}=Q_{I} p_{k}, \quad \text { for } 1 \leq k \leq K .
\end{gathered}
$$

Next, we define the sequence of rewards. The reward of stopping after observing $X_{n}$ is defined as the throughput achieved by transmitting over channel $c_{n}$ and with the entire time span (i.e., from the beginning of observing $X_{1}$ until the end of transmission over channel $c_{n}$ ) taken into account. Note that the use of channel must be non-recall. Mathematically, the reward for transmitting over channel $c_{n}$ is given by

$$
y_{n}\left(X_{1}, \ldots, X_{n}\right) \stackrel{\text { def }}{=} y_{n}\left(X_{n}\right)=\frac{B_{\text {eff }}(n)}{T_{\text {tot }}(n)}=\frac{X_{n} \tau_{t}\left(1-P_{\text {loss }}\right)}{n\left(\tau_{s}+\tau_{p}\right)+\tau_{t}}
$$

where $B_{\text {eff }}(n)$ is the number of collision-free data bits that can be transmitted over channel $c_{n}, T_{\text {tot }}(n)$ is the total time cost including channel search and transmission times, and $P_{\text {loss }}$ is the probability that channel $c_{n}$ is re-occupied by some returning PR during the CR's transmission, which leads to a collision that voids the CR's transmission. Defining the moment of sensing as the reference point, denote the forward recurrence time of the channel's idle period by the random variable $\tilde{\tau}_{0}$ and its pdf by $\tilde{f}_{0}$. We can calculate $P_{\text {loss }}$ as follows

$$
P_{\text {loss }}=\operatorname{Pr}\left\{\tilde{\tau}_{0}<\tau_{t}\right\}=\int_{0}^{\tau_{t}} \tilde{f}_{0}(t) d t .
$$

Following standard renewal theory analysis:

$$
\tilde{f}_{0}(t)=\frac{1-\int_{0}^{t} f_{0}(\tau) d \tau}{\int_{0}^{\infty} \tau f_{0}(\tau) d \tau}
$$

where $f_{0}$ is the pdf of the channel's idle period. For example, if $f_{0}$ is an exponential distribution with mean $\alpha$, then $\tilde{f}_{0}=f_{0}$ and $P_{\text {loss }}=1-e^{-\frac{\tau_{t}}{\alpha}}$. 
Define $\Psi=\left\{N: N \geq 1, E\left[T_{\text {tot }}(N)\right]<\infty\right\}$ as the set of all possible stopping rules. Our goal is to find an optimal stopping rule $N^{*} \in \Psi$ that maximizes the following rate-ofreturn objective function:

$$
\operatorname{maximize}_{N \in \Psi} \frac{E\left\{B_{e f f}(N)\right\}}{E\left\{T_{\text {tot }}(N)\right\}} .
$$

Clearly, because the CR decides after each observation whether or not to stop (according to some rule), the final stopping time $N$ is a random variable. Therefore, the number of bits that can be effectively transmitted at the stopping point, $B_{\text {eff }}(N)$, together with the time cost $T_{\text {tot }}(N)$, are both random variables related to $N$. This is in contrast to the $B_{\text {eff }}(n)$ and $T_{t o t}(n)$ in (8), where $n$ is a constant.

\section{OPTIMAL STOPPING RULE AND OPTI- MIZATION CONSIDERATIONS}

In this section, we first solve the maximum-rate-of-return problem (11) using optimal stopping theory. We then further examine the structure of our solution to address the optimization issues raised in Section 1.

\subsection{Throughput-optimal Stopping Rule}

To solve (11), we first consider a transformed version of the problem, whose reward sequence is defined by

$$
\begin{aligned}
w_{n} & =B_{\text {eff }}(n)-\lambda T_{\text {tot }}(n) \\
& =X_{n} \tau_{t}\left(1-P_{\text {loss }}\right)-\lambda\left[n\left(\tau_{s}+\tau_{p}\right)+\tau_{t}\right] .
\end{aligned}
$$

Following [6], if the parameter $\lambda$ is chosen such that the optimal expected reward of the transformed problem, i.e., $V^{*} \stackrel{\text { def }}{=} \sup _{N \in \Psi} E\left\{B_{\text {eff }}(N)-\lambda T_{\text {tot }}(N)\right\}$, is zero, then the optimal stopping rule $N^{*}$ of this transformed problem is also the optimal stopping rule of the original problem (11). In addition, the value of $\lambda$ that gives $V^{*}=0$, denoted as $\lambda^{*}$, is the maximum throughput in (11) achieved by the optimal stopping rule $N^{*}$. Applying this philosophy, we present the following results regarding the existence and solution of the optimal stopping rule for problem (11).

Theorem 1: An optimal solution to (11) exists. The maximum throughput $\lambda^{*}$ that is achieved by this optimal stopping rule is the solution of: $E\left\{\max \left(X_{n} \tau_{t}\left(1-P_{\text {loss }}\right)-\lambda^{*} \tau_{t}, 0\right)\right\}=$ $\lambda^{*}\left(\tau_{s}+\tau_{p}\right)$. The optimal stopping rule is given by $N^{*}=$ $\min \left\{n \geq 1: X_{n} \geq \frac{\lambda^{*}}{1-P_{\text {loss }}}\right\}$.

The proof of this theorem is presented in Appendix A. Regarding the calculation of the optimal throughput and the optimal stopping rule of (11), we have the following theorem: Theorem 2: $\lambda^{*}$ is unique.

The proof is given in Appendix B. For the particular discreterate CRs considered in our work, a fast numerical algorithm can be developed to calculate the exact $\lambda^{*}$ in at most $\mathcal{O}(K)$ time, where $K$ is the number of rates supported by the CR. Such an algorithm is based on the following observations. First, for the multi-rate system $X_{n} \in\left\{R_{0}, R_{1}, \ldots, R_{K}\right\}$, where $R_{0}=0<R_{1}<\ldots<R_{K}$, define $k^{*}$ to be the minimum integer that satisfies $R_{k^{*}} \geq \frac{\lambda^{*}}{1-P_{\text {loss }}}$. Obviously, $1 \leq k^{*} \leq K$. Using this notation, the equation $E\left\{\max \left(X_{n} \tau_{t}\left(1-P_{\text {loss }}\right)-\right.\right.$ $\left.\left.\lambda^{*} \tau_{t}, 0\right)\right\}=\lambda^{*}\left(\tau_{s}+\tau_{p}\right)$ can be written as

$$
\begin{array}{r}
\sum_{k=k^{*}}^{K}\left(R_{k} \tau_{t}\left(1-P_{\text {loss }}\right)-\lambda^{*} \tau_{t}\right) q_{k}=\lambda^{*}\left(\tau_{s}+\tau_{p}\right), \\
\text { subject to } R_{k^{*}-1}<\frac{\lambda^{*}}{1-P_{\text {loss }}} \leq R_{k^{*}}
\end{array}
$$

This gives a candidate solution for $\lambda^{*}$

$$
\begin{aligned}
& \lambda^{*}=\frac{\tau_{t}\left(1-P_{\text {loss }}\right) \sum_{k=k^{*}}^{K} R_{k} q_{k}}{\tau_{s}+\tau_{p}+\tau_{t} \sum_{k=k^{*}}^{K} q_{k}}, \\
& \text { subject to } R_{k^{*}-1}<\frac{\lambda^{*}}{1-P_{\text {loss }}} \leq R_{k^{*}}
\end{aligned}
$$

The range of values for $k^{*}$ is from 1 to $K$. Therefore, one can first enumerate all candidates of $\lambda^{*}$ according to (14) by setting $k^{*}=1, \ldots, K$, respectively, and then pick the one that satisfies the condition $R_{k^{*}-1}<\frac{\lambda^{*}}{1-P_{\text {loss }}} \leq R_{k^{*}}$. Theorem 2 guarantees that there is only one solution satisfying this condition. The particular $R_{k^{*}}$ under which the right $\lambda^{*}$ is obtained is the threshold rule that determines whether an idle channel is good enough to be used.

\subsection{Optimization Considerations}

\subsubsection{Impact of Probing Overhead}

In this section, we evaluate $\lambda^{*}$ as a function of the parameters $\tau_{s}$ and $\tau_{p}$. An examination of (14) reveals that $\lambda^{*}$ can be written as a segmented function. Specifically, for the $j$ th segment, $1 \leq j \leq K$, the value of $\lambda^{*}$ satisfies $R_{j-1}\left(1-P_{\text {loss }}\right)<\lambda^{*} \leq \bar{R}_{j}\left(1-P_{\text {loss }}\right)$. To satisfy this condition, it must be the case that

$$
R_{j-1}<\frac{\tau_{t} \sum_{k=j}^{K} R_{k} q_{k}}{\tau_{s}+\tau_{p}+\tau_{t} \sum_{k=j}^{K} q_{k}} \leq R_{j}
$$

After some mathematical manipulations, (15) leads to the following condition:

$\frac{\sum_{k=j}^{K} R_{k} q_{k}-R_{j} \sum_{k=j}^{K} q_{k}}{R_{j}} \leq \frac{\tau_{s}+\tau_{p}}{\tau_{t}}<\frac{\sum_{k=j}^{K} R_{k} q_{k}-R_{j-1} \sum_{k=j}^{K} q_{k}}{R_{j-1}}$.

The ratio $\eta \stackrel{\text { def }}{=} \frac{\tau_{s}+\tau_{p}}{\tau_{t}}$ represents the efficiency of the channel sensing/probing/access scheme. All the time factors in (16) are separated from the upper and lower bounds of the segment, allowing a neat partition of segments based on $\eta$. Following this thread, $\lambda^{*}$ can be explicitly written in the following segmented form:

$$
\lambda^{*}= \begin{cases}\lambda_{1}^{*}(\eta) & \text { for } \phi_{1} \leq \eta<\Phi_{1} \\ \vdots & \\ \lambda_{j}^{*}(\eta) & \text { for } \phi_{j} \leq \eta<\Phi_{j} \\ \vdots & \\ \lambda_{K}^{*}(\eta) & \text { for } \phi_{K} \leq \eta<\Phi_{K}\end{cases}
$$

where $\lambda_{j}^{*}(\eta) \stackrel{\text { def }}{=} \frac{\left(1-P_{l o s s}\right) \sum_{k=j}^{K} R_{k} q_{k}}{\eta+\sum_{k=j}^{K} q_{k}}, \phi_{j} \stackrel{\text { def }}{=} \frac{\sum_{k=j}^{K} R_{k} q_{k}-R_{j} \sum_{k=j}^{K} q_{k}}{R_{j}}$, and $\Phi_{j} \stackrel{\text { def }}{=} \frac{\sum_{k=j}^{K} R_{k} q_{k}-R_{j-1} \sum_{k=j}^{K} q_{k}}{R_{j-1}}, j=1, \ldots, K$. Because we are interested in determining whether the inclusion of probing leads to better performance, we can assume $\tau_{s}$ and $\tau_{t}$ to be fixed (we later discuss the case when $\tau_{s}$ is an optimization variable), and analyze the structure of $\lambda^{*}$ as a function of $\tau_{p}$. In this sense, $\eta$ becomes a one-to-one image of $\tau_{p}$.

Theorem 3: Given $\tau_{s}$ and $\tau_{t}$, the function $\lambda^{*}$ defined in (17) is a continuous and strictly mono-decreasing segmented function over the entire domain of $\eta$.

The proof is given in Appendix $\dot{\mathrm{C}}$. When probing is not used, the average throughput, denoted by $\lambda_{n o-p r o b i n g}$, can be derived as follows. First, the number of channels that are sensed until an idle channel is found follows a geometric distribution with parameter $Q_{I}$. So the average time cost for finding an idle channel is given by $T_{s} \stackrel{\text { def }}{=} \tau_{s} / Q_{I}$. Once an idle 
channel is found, the average data rate supported by that channel is $\bar{R}=\sum_{k=1}^{K} R_{k} p_{k}$. So $\lambda_{n o_{p} \text { robing }}$ is calculated as

$\lambda_{\text {no-probing }}=\frac{\left(1-P_{\text {loss }}\right) \tau_{t} \sum_{k=1}^{K} R_{k} p_{k}}{T_{s}+\tau_{t}}=\frac{\left(1-P_{\text {loss }}\right) \sum_{k=1}^{K} R_{k} q_{k}}{\eta^{\prime}+Q_{I}}$

where $\eta^{\prime} \stackrel{\text { def }}{=} \frac{\tau_{s}}{\tau_{t}}$. Given $\tau_{s}$ and $\tau_{t}, \lambda_{n o-p r o b i n g}$ is a constant. The equation $\lambda^{*}(\eta)=\lambda_{n o-p r o b i n g}$ must have a unique solution. This is because when $\tau_{t}=0$, the sensing/probing/access scheme is at least as good as the sensing/access scheme; whereas when $\tau_{t} \rightarrow \infty, \lambda^{*}(\infty)=0<\lambda_{\text {no-probing. Th }}$ property presented in Theorem 3 guarantees the existence of a unique positive intersection between $\lambda^{*}(\eta)$ and $\lambda_{n o-p r o b i n g}$. Therefore, the maximum acceptable $\tau_{p}$ that guarantees a throughput gain for sensing/probing/access scheme is given by

$$
\tau_{p}^{\max }=\tau_{t} \lambda^{*-1}\left(\lambda_{n o-\text { probing }}\right)-\tau_{s}
$$

where $\lambda^{*-1}(\cdot)$ denotes the inverse function of $\lambda^{*}(\eta)$. The significance of (19) is that it dictates when probing should be used for a given set of sensing/probing/access parameters.

\subsubsection{Impact of Sensing Time}

In this section, we are interested in the impact of $\tau_{s}$ on the optimal throughput. It is well known that for a given sensing/access CR system, throughput is a concave function of $\tau_{s}$ [11]. So there exists an optimal sensing time that maximizes the throughput. However, our finding in this section reveals that, in general, the concavity of the throughput is not preserved when probing is included, largely due to the more complicated structure of the multi-rate system. The encouraging aspect of our finding is that when $\tau_{s}$ is the variable, the throughput maintains its segmented structure. Treating a segment as our evaluation unit, the trend in throughput is concave over the segments of $\tau_{s}$. Based on this fact, we can derive a closed range $\mathbf{T}_{s}^{o}$ that contains the optimal sensing time $\tau_{s}^{o}$. The importance of this range is that any $\tau_{s} \in \mathbf{T}_{s}^{o}$ leads to a throughput greater than what can be achieved under any $\tau_{s} \notin \mathbf{T}_{s}^{o}$. The range $\mathbf{T}_{s}^{o}$ is also "provably efficient", i.e., any value inside this range can achieve at least a provable fraction of the maximum throughput achieved with $\tau_{s}^{o}$. As a result, achieving provably near-optimal performance is still guaranteed.

Our analysis involves evaluating the partition points of each segment defined in (17). In total, there are $K+1$ distinct partition points: $\phi_{0} \stackrel{\text { def }}{=} \Phi_{1}=\infty>\phi_{1}>\phi_{2}>\ldots>$ $\phi_{K-1}>\phi_{K}=0$, where the new notation $\phi_{0}$ is defined for presentation convenience. For $1 \leq j \leq K-1, \phi_{j}$ can be written as

$$
\phi_{j}=\left(1-P_{f a}\left(\tau_{s}\right)\right) C_{j}
$$

where $C_{j} \stackrel{\text { def }}{=} \frac{P_{I} \sum_{k=j}^{K}\left(R_{k}-R_{j}\right) p_{k}}{R_{j}}$ is a channel-dependent quantity that does not depend on $\tau_{s}$.

We consider an energy detector for channel sensing, for which the false alarm probability is approximated by [11]

$$
P_{f a}\left(\tau_{s}\right)=Q\left(\left(\frac{\epsilon}{\sigma_{u}^{2}}-1\right) \sqrt{\tau_{s} f_{s}}\right)
$$

where $\frac{\epsilon}{\sigma_{u}^{2}}$ is the decision threshold for sensing, $f_{s}$ is the channel bandwidth, and $Q($.$) is the Q-function. Given a tar-$ get minimum sensing time $\tau_{s}^{\text {min }}$ and a desired miss detection probability $\bar{P}_{m d}$, the decision threshold should be chosen such that for any $\tau_{s} \geq \tau_{s}^{\min }$, we have $P_{m d}\left(\tau_{s}\right) \leq \bar{P}_{m d}$, i.e., [11]

$$
\frac{\epsilon}{\sigma_{u}^{2}}=Q^{-1}\left(1-\bar{P}_{m d}\right) \sqrt{2 \gamma+1} \tau_{s}^{\min } f_{s}+\gamma+1
$$

where $\gamma$ is the received signal-to-noise ratio of the PR signal as measured at the CR. The relationship between $P_{f a}$ and $\tau_{s}$

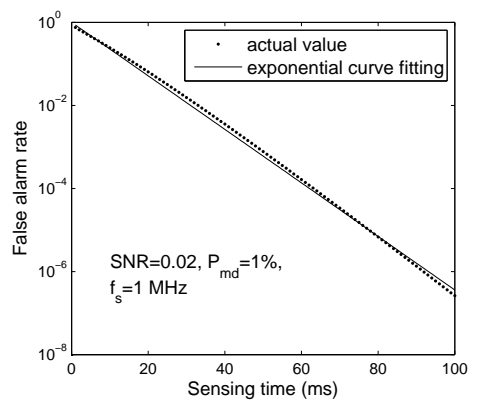

Figure 3: Exponential curve fitting of the false alarm rate.

in (21) is not in closed-form and thus is hard to manipulate. Given the parameters $\gamma, \tau_{s}^{\mathrm{min}}, P_{m d}$, and $f_{s}$, we suggest an exponential curve fitting for $(21)$, yielding $P_{f a}\left(\tau_{s}\right) \approx e^{-b \tau_{s}}$. Mathematically, this fitting is inspired by the well-known approximation [16] $\operatorname{erfc}(x) \leq e^{-x^{2}}$. Numerically, we found this exponential fitting to achieve good accuracy. Figure 3 shows an example when $\gamma=0.01, \tau_{s}^{\min }=0.1 \mathrm{~ms}, P_{m d}=1 \%$, and $f_{s}=1 \mathrm{MHz}$. The average fitting error in this case is less than $8 \%$.

Applying the exponential fitting of $P_{f a}\left(\tau_{s}\right)$ and treating $\tau_{s}$ as a variable, the domain of the $j$ th segment defined in (17) now becomes:

$$
\left(1-e^{-b \tau_{s}}\right) C_{j} \tau_{t}<\tau_{s}+\tau_{p} \leq\left(1-e^{-b \tau_{s}}\right) C_{j-1} \tau_{t} .
$$

The above partition is not in explicit form of $\tau_{s}$ because $\tau_{s}$ appears on both sides of each inequality. To get the explicit partitions, we need to solve the following set of equations of $\tau_{s}$ :

$$
\tau_{s}=\left(1-e^{-b \tau_{s}}\right) C_{j} \tau_{t}-\tau_{p}, \quad 1 \leq j \leq K
$$

For each equation, if a non-negative solution exists, then it gives a partition point over $\tau_{s}$. The difficulty here is that such a solution does not always exist.

Theorem 4: The following statements specify the existential condition and structure of the solutions to (24):

1. Existential condition: An equation in (24) has solutions if and only if $C_{j} \tau_{t}-\frac{1}{b}-\frac{1}{b} \ln b C_{j} \tau_{t} \geq 0$

2. Number of solutions: Each equation in (24) can have at most two solutions. At most one equation can have exactly one solution;

3. Sign of solutions: If an equation has two solutions, then both solutions are positive (or negative) if $\frac{1}{b} \ln b C_{j} \tau_{t}$ is positive (or negative). In other words, it is impossible to have one positive solution and one negative solution for the same equation;

4. Structure of solutions: If the $j$ th equation has two positive solutions, denoted as $\tau_{s}^{(j, \text { high })}$ and $\tau_{s}^{(j, \text { low })}$, where $\tau_{s}^{(j, \text { high })} \geq$ $\tau_{s}^{(j \text {,low })}>0$, then the $(j-1)$ th equation must have two positive solutions, which satisfy the condition $\tau_{s}^{(j-1, \text { high })}>$ $\tau_{s}^{(j, \text { high })} \geq \tau_{s}^{(j, \text { low })}>\tau_{s}^{(j-1, \text { low })}>0$.

To emphasize the structure of the solutions to (24), we present the proof in the body of the paper.

Proof: 1. We first prove the existential condition. Define

$$
h_{j}\left(\tau_{s}\right)=\left(1-e^{-b \tau_{s}}\right) C_{j} \tau_{t}-\tau_{p}-\tau_{s}, \quad 1 \leq j \leq K .
$$

The equation set $h_{j}\left(\tau_{s}\right)=0,1 \leq j \leq K$, is equivalent to $(24)$. The function $h_{j}$ is concave since its second-order derivative is:

$$
\frac{d^{2} h_{j}}{d \tau_{s}^{2}}=-b^{2} C_{j} \tau_{t} e^{-b \tau_{s}}<0 .
$$




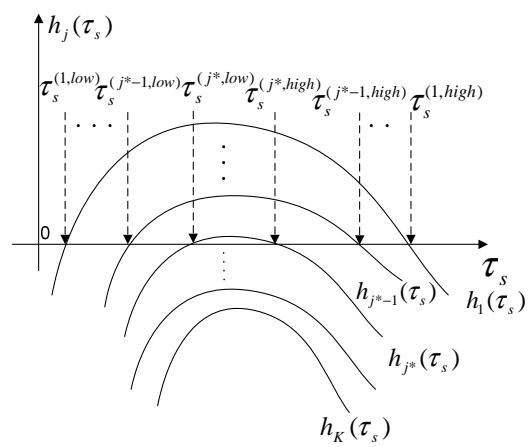

Figure 4: Structure of the solutions to (24) and the partition points over $\tau_{s}$.

Because of this concavity, it is clear that $h_{j}\left(\tau_{s}\right)=0$ has solutions if and only if the function's maximum value is not smaller than 0 . The maximum value is calculated as follows:

$$
\frac{d h_{j}}{d \tau_{s}}=e^{-b \tau_{s}} b C_{j} \tau_{t}-1=0 .
$$

From (27) we can get $\tau_{s}^{o}=\frac{1}{b} \ln b C_{j} \tau_{t}$. Accordingly, the maximum value of $h_{j}\left(\tau_{s}\right)$ is given by

$$
h_{j}^{\max } \stackrel{\text { def }}{=} h_{j}\left(\tau_{s}^{o}\right)=C_{j} \tau_{t}-\frac{1}{b}-\frac{1}{b} \ln b C_{j} \tau_{t}
$$

Then statement 1 follows.

2. The first half of statement 2 is clear due to the concavity of $h_{j}\left(\tau_{s}\right)$. We prove the second half after proving statement 4.

3. The proof is by contradiction. We first consider the case when $\tau_{s}^{o}=\frac{1}{b} \ln b C_{j} \tau_{t}>0$. From the concavity of $h_{j}$, it is clear that at least one solution must be positive. Now suppose the second solution is negative. Then $h_{j}(0) \geq 0$ must hold. However, from (25), when $\tau_{s}=0, h_{j}(0)=-\tau_{p}<0$. A similar contradiction can be established when $\tau_{s}^{o}=\frac{1}{b} \ln b C_{j} \tau_{t}<0$. So statement 3 holds.

4. From the definition of $C_{j}$ in $(20)$, it is clear that $C_{j}<$ $C_{j-1}$. Now consider the solution $\tau_{s}^{(j, \text { high })}$ of the $j$ th equation. From (24), we have

$$
\tau_{s}^{(j, \text { high })}=\left(1-e^{b \tau_{s}^{(j, \text { high })}}\right) C_{j} \tau_{t}-\tau_{p}<\left(1-e^{b \tau_{s}^{(j, \text { high })}}\right) C_{j-1} \tau_{t}-\tau_{p} .
$$

As $\tau_{s} \rightarrow \infty,\left(1-e^{-b \tau_{s}}\right) C_{j-1} \tau_{t}-\tau_{p} \rightarrow C_{j-1} \tau_{t}-\tau_{p}<\infty$. So the function $\left(1-e^{-b \tau_{s}}\right) C_{j-1} \tau_{t}-\tau_{p}$ must intersect with the function $\tau_{s}$ between $\tau_{s}^{(j, \text { high })}$ and $\infty$ (the two boundaries not included). Applying a similar logic to $\tau_{s}^{(j, \text { low })}$, it is clear that the function $\left(1-e^{-b \tau_{s}}\right) C_{j-1} \tau_{t}-\tau_{p}$ also intersects with the function $\tau_{s}$ between 0 and $\tau_{s}^{(j \text {,low) }}$ (the two boundaries not included $)$. Note that the domains $\left(\tau_{s}^{(j, \text { up })}, \infty\right)$ and $(0$, $\left.\tau_{s}^{(j, \text { low })}\right)$ do not overlap. Accounting for statements 2 and 3 , it can be concluded that there are only two solutions to the $(j-1)$ th equation, each of which is positive. One solution is located in $\left(\tau_{s}^{(j, \text { high })}, \infty\right)$ and the other in $\left(0, \tau_{s}^{(j, \text { low })}\right)$. So statement 4 follows.

Based on statement 4, the second half of statement 2 is straightforward: Counting down from $j=K$ to $j=1$ in (24), the first equation that has solutions, say the $j^{*}$ th one, is the only one that can have exactly one solution. For all $j<j^{*}$, each equation must have two solutions.

The structure of the solutions to (24) and the resulting partition points over $\tau_{s}$ are illustrated in Figure 4 . The optimization of $\tau_{s}$ is based on examining the structure of this segmentation. Specifically, counting down from $j=K$ to 1 in (24), let the $j^{*}$ th equation be the first one that has positive solution(s). The segmented function $\lambda^{*}\left(\tau_{s}\right)$ is described as follows: 1 . The total number of segments is $2 j^{*}+1$. 2 . The domains of these segments are given from left to right by $\left[0, \tau_{s}^{(1, \text { low })}\right),\left[\tau_{s}^{(1, \text { low })}, \tau_{s}^{(2, \text { low })}\right), \ldots,\left[\tau_{s}^{\left(j^{*}, \text { low }\right)}, \tau_{s}^{\left(j^{*}, \text { high }\right)}\right)$, $\left[\tau_{s}^{\left(j^{*}, \text { high }\right)}, \tau_{s}^{\left(j^{*}-1, \text { high }\right)}\right), \ldots,\left[\tau_{s}^{(1, \text { high })}, \infty\right) . \quad 3$. Recalling the condition (15) required for $\lambda^{*}$, for the $j$ th left-most and the $j$ th right-most segments, where $1 \leq j \leq j^{*}$, the corresponding $\lambda^{*}$ must satisfy $\left(1-P_{\text {loss }}\right) R_{j-1}<\lambda^{*} \leq\left(1-P_{\text {loss }}\right) R_{j}$. In addition, the specific value of $\lambda^{*}$ in these two segments is given by

$$
\lambda^{*}=\frac{\left(1-e^{-b \tau_{s}}\right) \tau_{t} P_{I}\left(1-P_{\text {loss }}\right) \sum_{k=j}^{K} R_{k} p_{k}}{\tau_{s}+\tau_{p}+\left(1-e^{-b \tau_{s}}\right) \tau_{t} P_{I} \sum_{k=j}^{K} p_{k}} .
$$

Three properties regarding this segmentation can be observed: First, inside each segment, the relationship between $\lambda^{*}$ and $\tau_{s}$, i.e., (30), is no longer convex or monotonic. So $\lambda^{*}$ is in general neither convex nor monotonic for the entire domain $\tau_{s} \geq 0$. Second, the trend of $\lambda^{*}$ is concave with $\tau_{s}$ if we treat segment as our observation unit: Starting from the left-most segment, $\left[0, \tau_{s}^{(1, \text { low })}\right)$, any $\tau_{s}$ in the next segment gives greater $\lambda^{*}$ than any $\tau_{s}$ in the previous segment. This trend is valid until reaching the segment in the middle, $\left[\tau_{s}^{\left(j^{*}, \text { low }\right)}, \tau_{s}^{\left(j^{*}, \text { high }\right)}\right)$. Starting from this segment and until the last one, any $\tau_{s}$ in the next segment gives smaller $\lambda^{*}$ than any $\tau_{s}$ in the previous segment. Third, the segment $\left[\tau_{s}^{\left(j^{*} \text {,low }\right)}, \tau_{s}^{\left(j^{*}, \text { high }\right)}\right)$ gives $\lambda^{*}$ s that are greater than any other segments. In other words, we can define $\mathbf{T}_{s}^{o} \stackrel{\text { def }}{=}\left\{\tau_{s}\right.$ : $\left.\left.\tau_{s}^{\left(j^{*}, \text { low }\right)} \leq \tau_{s} \leq \tau_{s}^{\left(j^{*}, \text { high }\right)}\right)\right\} . \mathbf{T}_{s}^{o}$ is a closed range that contains the optimal $\tau_{s}^{o}$. In addition, any $\tau_{s} \in \mathbf{T}_{s}^{o}$ achieves greater throughput than any $\tau_{s} \notin \mathbf{T}_{s}^{o}$, and its throughput is bounded by $\left(1-P_{\text {loss }}\right) R_{j^{*}} \leq \lambda^{*} \leq\left(1-P_{\text {loss }}\right) R_{j^{*}+1}$. Therefore, for the sensing/probing/access scheme, even though we cannot find $\tau_{s}^{o}$ explicitly, we can still decide a good range for $\tau_{s}$ that provably gives near-optimal performance.

Theorem 5: The closed range $\mathbf{T}_{s}^{o}$ is $\frac{R_{j^{*}}}{R_{j^{*}+1}}$-optimal, i.e., any $\tau_{s} \in \mathbf{T}_{s}^{o}$ can achieve at least $\frac{R_{j^{*}}}{R_{j^{*}+1}}$ fraction of the maximum throughput, where $j^{*}$ denotes the id of the first equation that has positive solution(s) when counting down from the $K$ th to the first equation in (24).

\section{THROUGHPUT ANALYSIS FOR CRNS}

In this section, we study the aggregate throughput when multiple close-by CRs share the same spectrum, each being driven by its own sensing/probing/access process discussed before. Such an investigation allows us to better appreciate the proposed scheme from a network's standpoint. An important factor we need to consider in this scenario is collisions between CRs, i.e., more than one CR transmitter/receiver pair are sensing and probing the same channel at the same time, so their probing packets collide. As a result, none of them can use the channel at this moment even if this channel is idle and is of a good quality.

We consider two sensing strategies for CRs: random sensing and collaborative sensing. In random channel sensing, each CR pair randomly selects a channel to sense in each step. There is no information exchange between different CR pairs. For collaborative sensing, CRs exchange their channelhopping information in every step to avoid multiple CRs hopping to the same channel at the same time.

To make the analysis tractable, we relax the condition that CR's transmission time has a fixed length $\tau_{t}$. We assume that the transmission time is exponentially distributed with mean $\tau_{t}$. In the simulation section, we test the validity of 
this assumption and show that it has a negligible impact on network performance. A discrete-time Markov-chain model is used to analyze the throughput of the CRN. Time is divided into slots with slot length $=\tau_{s}+\tau_{p}$. So for a CR, each step of channel sensing/probing takes exactly one slot and each transmission takes on average $L \stackrel{\text { def }}{=}\left\lceil\frac{\tau_{t}}{\tau_{s}+\tau_{p}}\right\rceil$ slots. We assume that CRs are synchronized, i.e., the slots of different CRs are aligned. Let the number of CR transmitter/receiver pairs be $M$. To simplify the presentation, here we only consider the fundamental case when each CR link can only sense, probe, and transmit over one channel at a time. The case that a CR link can simultaneously use $J>1$ channels can be treated as $J$ independent one-channel virtual CR links and analyzed accordingly. To evaluate the CRN's capability of harvesting the spectrum, we are interested in a saturated traffic scenario, i.e., there is always backlogged traffic at each CR link. The state of the Markov chain is defined by the tuple $\left(x_{1}, \ldots, x_{M}\right)$, where each element $x_{m} \in\{0,1\}$ indicates the activity of the $m$ th CR link in the current slot: $x_{m}=0$ means that CR link $m$ is sensing and probing a channel; $x_{m}=1$ denotes an ongoing transmission by that link. A CR link's activity in the current slot depends on the effect of all CR links' activities in the previous slot, $\left(x_{1}^{\prime}, \ldots, x_{M}^{\prime}\right)$, i.e., the transition probability of the chain satisfies

$$
\operatorname{Pr}\left(x_{1}, \ldots, x_{M} \mid x_{1}^{\prime}, \ldots, x_{M}^{\prime}\right)=\prod_{m=1}^{M} \operatorname{Pr}\left(x_{m} \mid x_{1}^{\prime}, \ldots, x_{M}^{\prime}\right)
$$

Without loss of generality, we consider the transition probability of CR link 1. We first consider the random sensing strategy. Our analysis covers the following four cases:

Case 1: $\operatorname{Pr}\left(0 \mid 0, x_{2}^{\prime}, \ldots, x_{M}^{\prime}\right)$

In this case, the transition probability contains four components

$$
\begin{aligned}
\operatorname{Pr}\left(0 \mid 0, x_{2}^{\prime}, \ldots, x_{M}^{\prime}\right)= & P_{\text {cr_occupied }}+P_{\text {cr_collision }}+P_{\text {pr_occupied }} \\
& +P_{\text {poor_channel }}
\end{aligned}
$$

where $P_{\text {cr_occupied }}$ denotes the probability that the channel that was sensed/probed by CR link 1 in the previous slot was being occupied (transmitted over) by other CR links, and thus CR link 1 has to sense/probe another channel in current slot; $P_{c r-c o l l i s i o n}$ denotes the probability that the channel that was sensed/probed by CR link 1 in the previous slot was not being occupied by any other CR, but was being sensed/probed by other CR links, and hence there was a collision and CR link 1 has to sense/probe another channel in the current slot; $P_{\text {pr_occupied }}$ denotes that the channel that was sensed/probed by CR link 1 was neither being occupied nor being sensed/probed by any other CR, but was being used by a PR, and hence CR link 1 has to sense/probe another channel in current slot; $P_{\text {poor_channel }}$ denotes that the channel that was sensed/probed by CR link 1 in the previous slot was neither being occupied by either CR or PR, nor being sensed/probed by any other CR, but it was having a bad channel condition, and hence CR link 1 has to sense/probe another channel in current slot.

To calculate these four probabilities, we note that a collision of multiple CRs that are sensing the same channel can be detected during the probing phase. After detecting a collision, the CRs will sense/probe other channels in the next slot. Therefore a CR's transmission over some channel indicates this channel is collision-free between CRs. We define the following notations: $M_{1}^{(m)} \stackrel{\text { def }}{=} \sum_{i=1 ; i \neq m}^{M} x_{i}^{\prime}$ and $M_{0}^{(m)} \stackrel{\text { def }}{=}(M-1)-M_{1}^{(m)} \cdot M_{1}^{(m)}$ denotes the number of transmitting CR links in the previous slot, not counting the $m$ th link; $M_{0}^{(m)}$ is the number of sensing/probing channels in the previous slot, not counting the $m$ th link. Then, we have

$$
P_{\text {croccupied }}=M_{1}^{(1)} / C
$$

$$
\begin{gathered}
P_{\text {cr_collision }}=\left(1-\frac{M_{1}^{(1)}}{C}\right)\left[1-\left(\frac{C-1}{C}\right)^{M_{0}^{(1)}}\right] \\
P_{\text {pr_occupied }}=\left(1-\frac{M_{1}^{(1)}}{C}\right)\left(\frac{C-1}{C}\right)^{M_{0}^{(1)}} Q_{B} \\
P_{\text {poor_channel }}=\left(1-\frac{M_{1}^{(1)}}{C}\right)\left(\frac{C-1}{C}\right)^{M_{0}^{(1)}} Q_{I} \sum_{k=0}^{k^{*}-1} p_{k}
\end{gathered}
$$

Case 2: $\operatorname{Pr}\left\{1 \mid 0, x_{2}^{\prime}, \ldots, x_{M}^{\prime}\right\}$

This transition probability is simply given by

$$
\begin{aligned}
& \operatorname{Pr}\left\{1 \mid 0, x_{2}^{\prime}, \ldots, x_{M}^{\prime}\right\}=1-\operatorname{Pr}\left\{0 \mid 0, x_{2}^{\prime}, \ldots, x_{M}^{\prime}\right\} \\
= & \left(1-\frac{M_{1}^{(1)}}{C}\right)\left(\frac{C-1}{C}\right)^{M_{0}^{(1)}} Q_{I} \sum_{k=k^{*}}^{K} p_{k}
\end{aligned}
$$

Case 3: $\operatorname{Pr}\left\{0 \mid 1, x_{2}^{\prime}, \ldots, x_{M}^{\prime}\right\}$

This case means that CR link 1 finishes its transmission in the previous slot, so it starts looking for a new channel in the current slot. The transition probability is given by

$$
\operatorname{Pr}\left\{0 \mid 1, x_{2}^{\prime}, \ldots, x_{M}^{\prime}\right\}=1 / L
$$

Case 4: $\operatorname{Pr}\left\{1 \mid 1, x_{2}^{\prime}, \ldots, x_{M}^{\prime}\right\}$

This is simply calculated as

$$
\operatorname{Pr}\left\{1 \mid 1, x_{2}^{\prime}, \ldots, x_{M}^{\prime}\right\}=1-\operatorname{Pr}\left\{0 \mid 1, x_{2}^{\prime}, \ldots, x_{M}^{\prime}\right\}=(L-1) / L .
$$

Having obtained the transition probability matrix, the Markov chain's stationary distribution for the random vector $\left(x_{1}, \ldots, x_{M}\right)$ can be calculated using standard state-transition balance equations. Among all the states, those with $\sum_{m=1}^{M} x_{i}>C$ are infeasible, and therefore their stationary distribution probability is 0 . The CRN's throughput is then calculated as

$$
R_{\text {tot }}=\sum_{x_{1}=0}^{1} \ldots \sum_{x_{M}=0}^{1}\left(\operatorname{Pr}\left\{x_{1}, \ldots, x_{M}\right\} \sum_{m=1}^{M} x_{m} \bar{R}\right)
$$

where $\bar{R} \stackrel{\text { def }}{=} \frac{\left(1-P_{l o s s}\right) \sum_{k=k^{*}}^{K} R_{k} p_{k}}{\sum_{k=k^{*}}^{K} p_{k}}$ is the average throughput a CR link can achieve when transmitting.

In the above calculation, we have assumed a fully-distributed random channel sensing strategy. Under a collaborative sensing strategy, the above calculation should be modified as follows: the term $\left(\frac{C-1}{C}\right)^{M_{0}^{(1)}}$ should be replaced by $\left(\frac{C-1}{C}\right)^{\max \left(0, M_{0}^{(1)}+1-C\right)}$ in (33) through (37). This is because under collaborative sensing, if the number of links that are sensing channels is not greater than the number of channels, then there is no collision. Otherwise, the collision is only due to those links that exceed the number of channels.

\section{PERFORMANCE EVALUATION}

In this section, we use simulations to evaluate the performance of the proposed sequential channel sensing/probing scheme. Our simulations are developed using CSIM programs (CSIM is a C-based process-oriented discrete-event simulation package). The simulation results presented below are based on the average of ten independent runs, each lasting for 500 seconds of simulation time.

\subsection{Simulations for a Single CR link}

We first study the performance of a single CR link as a function of various operational parameters. This scenario is meant to verify the accuracy of our analytical model and illustrate the significance of our optimization. We are interested in the following performance metrics: (1) the average 


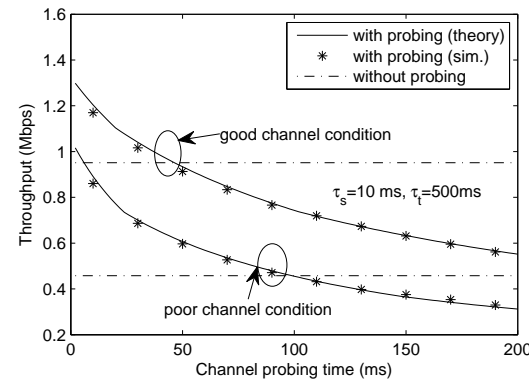

Figure 5: Throughput vs. probing time.

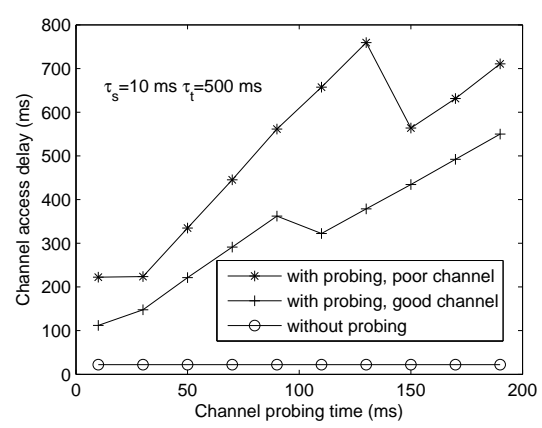

probing time.

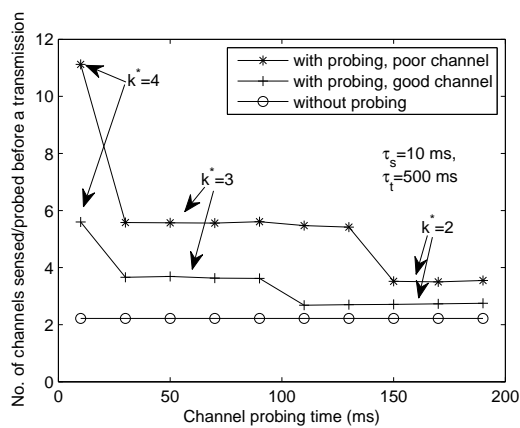

Figure 7: Number of sensed/probed channel before a transmission vs. probing time. throughput, defined as the average number of bits transmitted by the CR link in each second; (2) the average channel access delay, defined as the average time the CR spends until it finds a good channel and starts transmitting over it; and (3) the average number of sensed/probed channels before a transmission takes place. Note that although the average throughput is the primary objective of our optimization, the delay-related metrics (2) and (3) carry some significance due to the sequential natural of the scheme.

We simulate a CR link that supports five rates: $0,1,2,3$, and $4 \mathrm{Mbps}$. We consider the rate distribution, $\left(p_{0}, p_{1}, p_{2}, p_{3}, p_{4}\right)$ under two channel conditions: a good channel condition with rate distribution $(0.1,0.1,0.2,0.2,0.4)$ and a poor channel condition with rate distribution $(0.4,0.2,0.2,0.1,0.1)$. We assume that the rate distributions over various channels are i.i.d. and that the fluctuation over a channel is quasi-static, i.e., the maximum rate supported by the channel remains unchanged during the CR's transmission time. We set $\tau_{t}=500$ ms, and the average idle and busy period of each channel $\alpha=\beta=500 \mathrm{~ms}$

In Figures 5 to 7 , we study the performance as a function of the channel probing time. The channel sensing time is fixed at $10 \mathrm{~ms}$. We take $P_{f a}=0.1$. To verify the accuracy of our analysis, the throughput calculated according to the analytical model and the one obtained from the simulations are compared in Figure 5. The throughput in the absence of probing is also plotted. We observe that the simulated throughput matches well the one obtained analytically. Furthermore, it is clear that as long as $\tau_{p}$ is kept sufficiently small, e.g., $\tau_{p}<45 \mathrm{~ms}$ under a good channel condition or $\tau_{p}<100 \mathrm{~ms}$ under a poor channel condition, the incorporation of probing leads to a throughput gain. Recalling that the per-channel probing time in current 802.11 WLAN systems ranges from 10 to $133 \mathrm{~ms}$ [1], the above probing time requirement is non-trivial, because there is still a reasonable chance under the current technology that the use of probing could actually undermine the throughput. In addition, the benefit of probing is more significantly observed under poor channel conditions, e.g., the throughput gain reaches about $120 \%$ when $\tau_{p}=10 \mathrm{~ms}$. At the same time, the maximum acceptable channel probing time becomes $100 \mathrm{~ms}$. These results favor the use of probing when the channel condition is bad, which is in line with our intuition.

Figure 6 shows that significant channel access delay is caused by the sequential channel sensing and probing. Such delay is expected, because as shown in Figure 7, the CR needs to sense and probe more channels to find an idle one that also meets the probing threshold. (Note that this delay has been accounted for in calculating the throughput in Figure 5.) The long channel access delay also suggests that the proposed scheme is most suitable for non-realtime applications, which can tolerate certain delay in exchange of higher throughput.
In addition, we also observe from Figure 7 that the larger the $\tau_{p}$, the smaller the number of channels that are scanned before a transmission. This is because as $\tau_{p}$ increases, so does the cost of scanning each channel. The increased cost of scanning a channel will eventually cancel out the benefit of finding a better channel via scanning more channels, and thus leading to smaller number of channels scanned before a transmission. The reduced number of scanned channels also leads to a smaller probing threshold. For example, as shown in Figure 7, under poor channel conditions, the rate threshold , becomes 4,3 , and 2 , for $\tau_{p} \leq 20 \mathrm{~ms}, 20 \mathrm{~ms}<\tau_{p} \leq 140 \mathrm{~ms}$, and $140 \mathrm{~ms}<\tau_{p} \leq 200 \mathrm{~ms}$, respectively. It is these switches in the rate threshold that incur the non-smooth change of delay observed in Figure 6.

The performance as a function of the channel sensing time is shown in Figures 8 to 10 . Here we use the exponential curve fitting with parameter $b=14.8349$ to describe the $P_{f a}$ vs. $\tau_{s}$ relationship. The theoretical and simulated throughput results are plotted in Figure 8. It is clear from this figure that the sensing time has a significant impact on the CR's throughput. For example, under good channel conditions, the ratio between the highest and the lowest throughput observed in the simulation can be as high as $190 \%$. This wide range supports the need to optimize the sensing time. In addition, the concave trend in the throughput curve is clearly observed in this figure. More importantly, even though we cannot analytically derive the globally optimal $\tau_{s}^{o}$ that maximizes the throughput, our analysis in Section 4 shows that they must be located in the ranges denoted by the dotted boxes, i.e., $\tau_{s}^{o} \in[15.1,43.9] \mathrm{ms}$ under good channel conditions, and $\tau_{s}^{o} \in[6.8,140.5] \mathrm{ms}$ under poor channel conditions. In practice, the sensing time should be selected from these ranges to achieve near-optimal throughput.

Figures 9 and 10 demonstrate the tradeoff between sensing time and sensing accuracy. More specifically, when $\tau_{s}$ is small, the CR needs to scan many channels before each transmission due to its poor sensing accuracy. The accumulated scanning time over these channels causes excessive channel access delay. As $\tau_{s}$ increases, the $\mathrm{CR}$ can more accurately detect each spectrum opportunity, requiring the scanning of fewer channels before a transmission. This mono-decreasing relationship between the number of scanned channels and $\tau_{s}$ is seen in Figure 10.

\subsection{Sensitivity Analysis}

In this section, we study the performance of the proposed scheme when there is uncertainty, e.g., errors or fluctuations, in the CR's working environment. Such uncertainty captures deviations from the nominal setup used in the optimization, and thus the optimized parameters may not be actually optimal in practice. We are interested in the performance gap between the nominal setting and the one that uses exact 


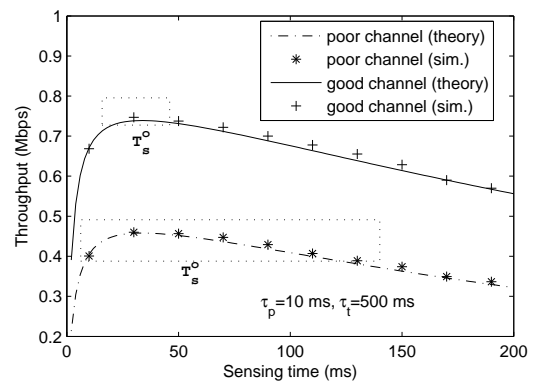

Figure 8: Throughput vs. sensing time.

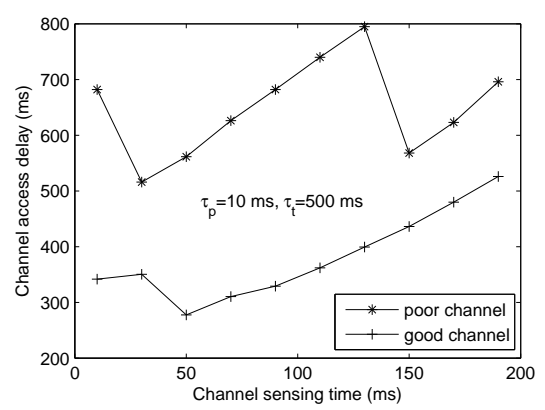

Figure 9: Char
sensing time.

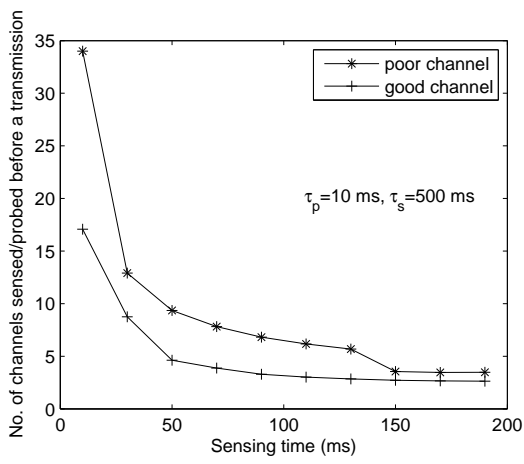

Figure 10: Number of scanned channels before a transmission vs. sensing time.

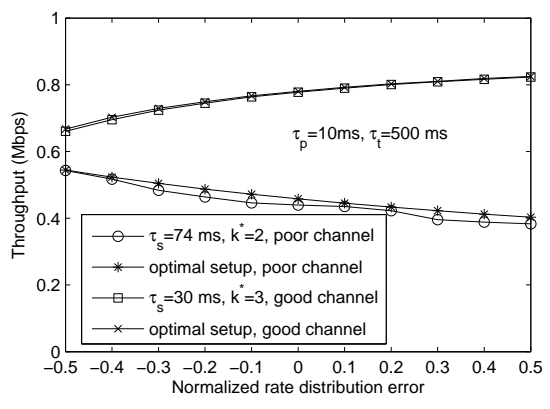

Figure 11: Throughput vs. rate distribution error.

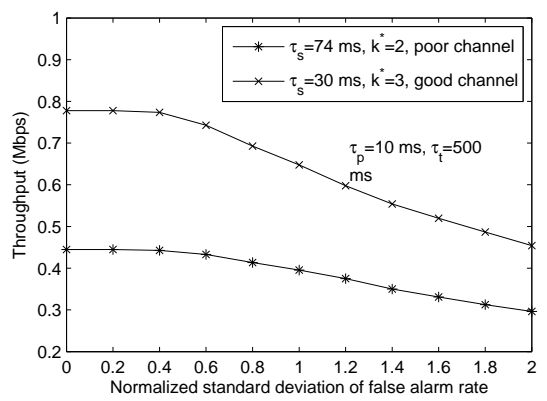

Figure 12: Throughput vs. error of channel sensing false alarm rate.

knowledge of the environment as input to the optimization. In particular, we focus on the uncertainty in the following two factors: channel's rate distribution $\left(p_{0}, \ldots, p_{K}\right)$ and the relationship between sensing accuracy and sensing time. Uncertainty in the rate distribution arises when the online measurement is not accurate due to, for example, a limited observation window. Uncertainty also happens when the actual distribution shifts with time. Similar situations apply to the $P_{f a}$ vs. $\tau_{s}$ relationship. Furthermore, this relationship also suffers from intrinsic curve-fitting errors.

We study the impact of rate-distribution uncertainty on throughput in Figure 11. We use the following model to describe uncertainty in the rate distribution. The distributions $(0.4,0.2,0.2,0.1,0.1)$ and $(0.1,0.1,0.2,0.2,0.4)$ are taken as the nominal rate distributions for the poor and good channel conditions, respectively. An actual rate distribution is generated according to a distribution error, $\delta$, in the following way. Under poor channel conditions, for a given distribution error $\delta$, the corresponding actual rate distribution is given by $\frac{1}{0.6(1+\delta)+0.4}(0.4(1+\delta), 0.2(1+\delta), 0.2,0.1,0.1)$. Under good channel conditions, the actual rate distribution is given by $\frac{1}{0.6(1+\delta)+0.4}(0.1,0.1,0.2,0.2(1+\delta), 0.4(1+\delta))$. In this way, an actual rate distribution literally differs from the nominal one, but still retains the basic pattern that makes it in line with good (or bad) channel conditions. For example, under bad channel conditions, when $\delta=-0.5$ and 0.5 , the corresponding actual rate distributions are $(0.2857$, $0.1429,0.2857,0.1429,0.1429)$ and $(0.4615,0.2308,0.1538$, $0.0769,0.0769)$, respectively, for which the low rates still occur with relatively high probabilities (and thus representing bad channel). Under each channel condition, we first conduct optimization based on the nominal rate distribution to derive the theoretically optimal operational parameters. For example, under the poor channel condition, we found that $\tau_{s}^{o} \in[6.8,140.5] \mathrm{ms}$. So we use the middle point $\tau_{s}=74$ $\mathrm{ms}$ and the corresponding rate threshold $k^{*}=2$ to drive the sequential channel sensing and probing process in our simulation. During the simulation, channel conditions are generated according to the actual rate distribution. For each actual rate distribution, the optimal throughput is also decided by exhaustively testing various combinations of operational parameters via simulation. Figure 11 depicts throughput achieved by using the nominal distribution as input to the optimization compared with the actual optimal throughput obtained via exhaustive testing. It is clear that the gap between these two are minor (less than $5 \%$ in the worst case). This observation suggests that our optimization framework is insensitive to rate distribution errors, and thus the operational parameters derived from our analytical model still achieve good performance in actual environments.

We study the impact of sensing inaccuracy in Figure 12. To model the uncertainty in the false alarm rate of the channel sensing process, we modify the exponential curve-fitting function into $P_{f a}\left(\tau_{s}\right)=(1+|\theta|) e^{-b \tau_{s}}$, where $\theta$ is a random variable that follows a normal distribution $N\left(0, \sigma^{2}\right)$. The parameter $\sigma$ denotes the standard deviation of the error in the false alarm rate normalized by the deterministic component $e^{-b \tau_{s}}$. Here, we take the absolute value of the error. This leads to false alarm rates that are always larger than the deterministic component. The simulation results gathered under this setup can be considered as a lower bound on the performance when both positive and negative errors on false alarm rate can happen. From Figure 12, the throughput is shown to degrade with the magnitude of the error. However, the throughput shows some tolerance to errors when their magnitudes are limited. For example, no obvious throughput degradation is observed when $\sigma$ is smaller than 0.4 and 0.6 under good and poor channel conditions, respectively. Recalling that the exponential fitting induces less than $8 \%$ fit- 
ting errors, the proposed framework presents enough margin to accommodate those errors without significantly impacting the effectiveness of the optimization.

\subsection{Simulations for A CR Network}

In this set of simulations, we use the optimal threshold derived for individual links to drive the operation of the CRN. In Figures 13, we fix the number of CR links $M=8$ and plot the CRN throughput as a function of the number of channels, $C$. The rate distribution for each $\mathrm{CR}$ link is given by $(0.2$, $0.2,0.2,0.2,0.2)$. We set $\tau_{s}=\tau_{t}=10 \mathrm{~ms}$, and $\tau_{t}=500 \mathrm{~ms}$. Both simulation and analytical results are plotted. Note that in the analysis we have assumed exponentially distributed transmission time, but in the simulation a CR's transmission time has a fixed length. Figure 13 shows that as long as the mean transmission time remains the same, the distribution of the transmission time only has a minor impact on the throughput, as evidenced by the good match between the analytical and the simulation results.

From Figure 13, it is also clear that the collaborative sensing strategy achieves higher throughput than the random sensing strategy, mainly due to the former's smaller collision probability. Under both strategies, the throughput increases with $C$. However, the rate of increase is fast when $C$ is small, and slow when $C$ is large. This trend is due to the smaller collision probability and the more likelihood of finding an idle channel when $C$ is large. A third observation is that when $C$ is large the slope of the throughput curve in the case of collaborative sensing is less steep than in the random sensing case. This is because the collision probability under collaborative sensing approximates zero when $C$ is large, and therefore the throughput increase is only due to the increased probability of finding a good idle channel.

In Figures 14 and 15 we compare the sequential channel sensing/probing scheme with the candidate-set-based channel selection scheme [12]. As discussed in Section 2, the main limitation of this scheme is the overlap between the sets of candidate channels pre-computed by various CRs. We simulate two scenarios: light overlap, where each candidate set, which is of size 5, contains 2 network-wide common channels, and high overlap, where there are 4 network-wide common channels in each candidate set. The remaining channels in a set are randomly picked from the pool that does not include the network-wide common channels. Such a setup mimics the realistic situation where CRs are located close to each other, and thus may sense similar channel conditions. Various channels differ in their statistical quality. However, the difference is slight. Otherwise, various candidate sets would completely overlap. To conduct a fair comparison, we assume that within a set of candidate channels, a CR could only sequentially sense/probe each channel, just as in the sequential sensing/probing scheme. From Figure 14, it can be observed that when the number of CRs in the system is low, there is no significant difference in the throughput between the two schemes. However, as we increase the number of CRs, less throughput can be achieved under the candidate-set-based scheme. The gap becomes even larger in the high-overlap scenario. The worse performance of the candidate-set-based scheme is because the overlap between the sets of candidate channels leads to high collisions during channel probing and access, especially when the number of CRs is large.

In Figure 15, we study the impact of the size of the candidate set on the CRN throughput. In this simulation, the number of common channels in a candidate set is taken as the smallest integer that is greater than or equal to $C$ times the fraction of common channels ( 0.4 or 0.7 , corresponding to low and high overlap considered in the simulation). In general, the throughput achieved under the candidate-set based scheme increases with the size of the candidate set, because of the smaller collision between channel probing and access.
Ultimately, when the candidate set contains all the channels, both schemes achieve the same throughput, because the candidate-set-based scheme actually reduces to the sequential channel sensing/probing scheme at this point.

\section{CONCLUSIONS}

Our study has indicated that a carefully designed joint channel sensing/probing scheme for CRNs can achieve significant throughput gains over the conventional mechanism that uses sensing alone. Our findings include: (1) the throughputoptimal probing strategy has a threshold structure, which basically determines whether a probed idle channel is good or bad, (2) to achieve throughput gain over the conventional sensing approach, the probing time has to be smaller than a certain value; otherwise using sensing alone can achieve better throughput, (3) when probing is used, the throughput in general is no longer a concave function of the sensing time, largely due to the more complicated multi-rate structure induced by the inclusion of probing. However, this function has a segmented structure. If we treat segments as our observation instances, the trend in this function is concave. We exploited this property to derive a range of values for the sensing time that provides provably near-optimal throughput performance.

\section{REFERENCES}

[1] W. Arbaugh. Improving the latency of the probe phase during 802.11 handoff, [online] available: http://www.umiacs.umd.edu/partnerships/ltsdocs/ arbaug_talk2.pdf.

[2] N. B. Chang and M. Liu. Optimal channel probing and transmission scheduling for opportunistic spectrum access. In Proceedings of the ACM MobiCom Conference, 2007.

[3] N. B. Chang and M. Liu. Competitive analysis of opportunistic spectrum access strategies. In Proceedings of the IEEE INFOCOM Conference, pages 2207-2215, Apr. 2008.

[4] Y. Chen, Q. Zhao, and A. Swami. Joint design and separation principle for opportunistic spectrum access in the presence of sensing errors. IEEE Transactions on Information Theory, 54(5):2053-2071, May 2008.

[5] Y. Chen, Q. Zhao, and A. Swami. Distributed spectrum sensing and access in cognitive radio networks with energy constraint.
IEEE Transactions on Signal Processing, 57(2):783-797, Feb. $I E E E$
2009.

[6] T. Ferguson. Optimal stopping and applications. Available online at http://www.math.ucla.edu/ tom/stopping/content.html.

[7] S. Guha, K. Munagala, and S. Sarkar. Optimizing transmission rate in wireless channels using adaptive probes. In $A C M$ Sigmetrics/Performance Conference, 2006. Poster paper.

[8] A. T. Hoang and Y. C. Liang. Adaptive scheduling of spectrum sensing periods in cognitive radio netwroks. In Proceedings of the IEEE GLOBECOM Conference, pages 3128-3132, 2007.

[9] Z. Ji, Y. Yang, J. Zhou, M. Takai, and R. Bagrodia. Exploiting medium access diversity in rate adaptive wireless LANs. In Proceedings of the ACM MobiCom Conference, pages 345-359, Sept. 2004.

[10] W. Y. Lee and I. F. Akyildiz. Optimal spectrum sensing framework for cognitive radio networks. IEEE Transactions on Wireless Communications, 7(10):3845-3857, Oct. 2008

[11] Y. C. Liang, Y. Zeng, E. C. Y. Peh, and A. T. Hoang. Sensing-throughput tradeoff for cognitive radio networks. IEEE Transactions on Wireless Communications, 7(4):1326-1337, Apr. 2008.

[12] A. W. Min and K. G. Shin. Exploiting multi-channel diversity in spectrum-agile networks. In Proceedings of the IEEE INFOCOM Conference, pages 56-60, Apr. 2008.

[13] J. Mitola. Cognitive radio: an integrated agent architecture for software defined radio. PhD Dissertation, RTH, Sweden, 2000.

[14] T. S. Rappaport. Wireless Communications: Principles $\mathcal{E}$ Practice. Prentice Hall, 2002.

[15] A. Sabharwal, A. Khoshnevis, and E. Knightly. Opportunistic spectral usage: bounds and a multi-band CSMA/CA protocol. IEEE/ACM Transactions on Networking, 15(3):533-545, June 2007.

[16] J. M. Wozencraft and I. M. Jacobs. Principles of Communication Engineering. John Wiley \& Sons, Inc, 1965.

[17] Q. Zhao and B. M. Sadler. A survey of dynamic spectrum access: signal processing, networking, and regulatory policy. IEEE Signal Processing Magazine, 24(3):79-89, 2007. 


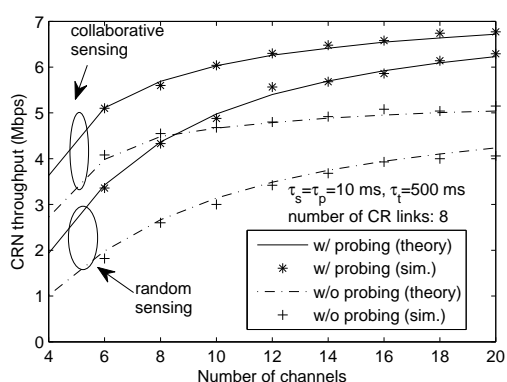

Figure 13: CRN throughput vs. Figure 14: CRN throughput vs. number of channels.

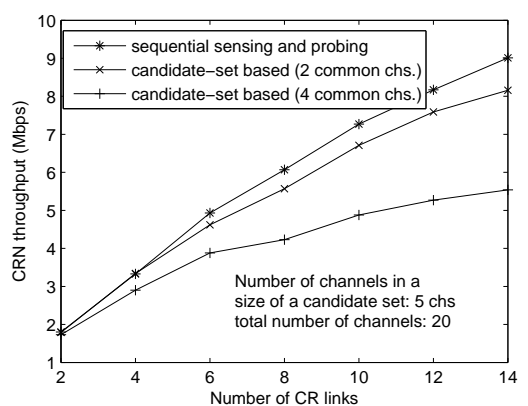

number of CR links.

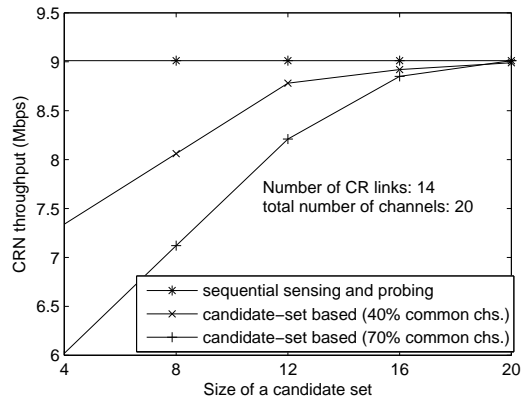

Figure 15: CRN throughput vs. size of candidate-channel set.
[18] Q. Zhao, L. Tong, A. Swami, and Y. Chen. Decentralized cognitive MAC for opportunistic spectrum access in ad hoc networks: a POMDP framework. IEEE Journal on Selected Areas in Communications, 25(3):589-600, Apr. 2007.

[19] D. Zheng, M. Cao, J. Zhang, and P. R. Kumar. Channel aware distributed scheduling for exploiting multi-receiver diversity and multiuser diversity in ad-hoc networks: a unified phy $/ \mathrm{mac}$ approach. In Proceedings of the IEEE INFOCOM Conference, pages 1454-1462, 2008.

[20] D. Zheng, W. Ge, and J. Zhang. Distributed opportunistic scheduling for ad-hoc communications: an optimal stopping approach. In Proceedings of ACM MobiHoc Conference, 2007.

[21] D. Zheng and J. Zhang. Joint optimal channel probing and transmission in collocated wireless networks. In Proceedings of the IEEE INFOCOM Conference, pages 2266-2270, May 2007.

\section{APPENDIX}

\section{A. PROOF OF THEOREM 1}

1. Existence. We need to prove that for any finite $\lambda$, an optimal stopping rule exists for the transformed problem (12). It follows from Theorem 1 in Chapter 3 of [6] that the optimal stopping rule exists if the following two conditions are satisfied:

1. $E\left\{\sup _{n} w_{n}\right\}<\infty$.

2. $\lim _{n \rightarrow \infty} \sup _{n} w_{n}=-\infty$, a.s.

By examining (12), condition 2 is clearly satisfied. Condition 1 can be verified by applying Theorem 1 in Chapter 4 of [6]. Specifically, it is easy to see that the random variable

$$
X_{n}^{\prime} \stackrel{\text { def }}{=} X_{n} \tau_{t}\left(1-P_{l o s s}\right)-\lambda \tau_{t}
$$

is related only to the random variable $X_{n}$. Because $X_{n}$ 's are i.i.d. for $n=1,2, \ldots$, the $X_{n}^{\prime}$ 's must also be i.i.d.. In addition, because $X_{n}$ takes a finite number of values and $\lambda$ is finite, $X_{n}^{\prime}$ must also be finite. Therefore, it holds that $E\left\{\max \left(X_{n}^{\prime}, 0\right)\right\}<\infty$ and $E\left\{\left(\max \left(X_{n}^{\prime}, 0\right)\right)^{2}\right\}<\infty$. So according to Theorem 1 in Chapter 4 of [6], it holds that $E\left\{\sup _{n} w_{n}\right\}=$ $E\left\{\sup _{n} X_{n}^{\prime}-n \lambda\left(\tau_{s}+\tau_{p}\right)\right\}<\infty$. So condition 1 is satisfied.

2. Optimal solution. $w_{n}$ can be written as $w_{n}=X_{n}^{\prime}-$ $n \lambda\left(\tau_{s}+\tau_{p}\right)$, where $X_{n}^{\prime}$ is defined in (41). So $X_{n}^{\prime}$ can be considered as the reward for observing $w_{1}, \ldots, w_{n}$, and $\lambda\left(\tau_{s}+\right.$ $\left.\tau_{p}\right)$ can be deemed as the cost for each observation. Applying the principle of optimality in Chapter 2 of [6], the optimal stopping rule of the transformed problem (12) is given by

$$
N^{*}=\min \left\{n \geq 1: X_{n}^{\prime} \geq V^{*}\right\}
$$

where $V^{*}$ denotes the expected return from an optimal stopping rule; it satisfies the following optimality equation

$$
V^{*}=E\left\{\max \left\{X_{n}^{\prime}, V^{*}\right\}\right\}-\lambda\left(\tau_{s}+\tau_{p}\right) .
$$

Equivalently, the above equation can be written as

$$
E\left\{\max \left(X_{n}^{\prime}-V^{*}, 0\right)\right\}=\lambda\left(\tau_{s}+\tau_{p}\right) .
$$

Recalling the connection between the original problem and its transformed version, the value of $\lambda$ that gives $V^{*}=0$ is simply the solution of (11). With $V^{*}=0$, we have the following equation:

$$
E\left\{\max \left(X_{n} \tau_{t}\left(1-P_{\text {loss }}\right)-\lambda^{*} \tau_{t}, 0\right)\right\}=\lambda^{*}\left(\tau_{s}+\tau_{p}\right) .
$$

According to Theorem 1 in Chapter 6 of [6], the solution to the above equation, $\lambda^{*}$, is the maximum objective function value for problem (11). At the same time, substituting $V^{*}=$ 0 into (42), we derive the optimal stopping rule to problem (11):

$$
N^{*}=\min \left\{n \geq 1: X_{n} \geq \frac{\lambda^{*}}{1-P_{\text {loss }}}\right\}
$$

This concludes the proof of Theorem 1 .

\section{B. PROOF OF THEOREM 2}

The main idea is to show that the LHS of (45) is a monodecreasing function in $\lambda^{*}$, whereas the RHS is mono-increasing in $\lambda^{*}$. The two functions must intersect at one and only one point. Particularly, we define $g(\lambda)=E\left\{\max \left(X_{n} \tau_{t}(1-\right.\right.$ $\left.\left.\left.P_{\text {loss }}\right)-\lambda \tau_{t}, 0\right)\right\}$. For the general case of a continuous random variable $X_{n}$ with pdf $q(x), g(\lambda)$ can be extended as

$$
g(\lambda)=\int_{\frac{\lambda}{1-P_{\text {loss }}}}^{\infty} x \tau_{t}\left(1-P_{\text {loss }}\right) q(x) d x-\int_{\frac{\lambda}{1-P_{\text {loss }}}}^{\infty} \lambda \tau_{t} q(x) d x .
$$

The first-order derivative of $g$ is given by

$$
\begin{aligned}
\frac{d g(\lambda)}{d \lambda}= & -\lambda \tau_{t} q\left(\frac{\lambda}{1-P_{\text {loss }}}\right) \\
& -\left[\tau_{t} \int_{\frac{\lambda}{1-P_{\text {loss }}}}^{\infty} q(x) d x-\lambda \tau_{t} q\left(\frac{\lambda}{1-P_{\text {loss }}}\right)\right] \\
= & -2 \lambda \tau_{t} q\left(\frac{\lambda}{1-P_{\text {loss }}}\right)-\tau_{t} \int_{\frac{\lambda}{1-P_{\text {loss }}}}^{\infty} q(x) d x
\end{aligned}
$$

Clearly, both terms in (48) are strictly negative, and therefore $g(\lambda)$ is a strictly mono-decreasing function. In addition, $g(0)=\tau_{t}\left(1-P_{\text {loss }}\right) E\left[X_{n}\right]<\infty$ and $g(\infty)=0$. It is clear that the RHS of (45) is a strictly mono-increasing function of $\lambda$ with function values of 0 and $\infty$ when $\lambda=0$ and $\lambda=\infty$, respectively. For the case of discrete random variable $X_{n}$, it is easy to see that the above monotonic property does not change. Therefore, $\lambda^{*}$ must have a unique solution.

\section{PROOF OF THEOREM 3}

First, by evaluating $\lambda_{j}^{*}(\eta)$, it is clear that inside each segment, $\lambda^{*}$ is continuous and strictly mono-decreasing. Next, it can be easily verified that $\Phi_{j}=\phi_{j-1}$ and $\lim _{\eta \rightarrow \Phi_{j}} \lambda_{j}^{*}(\eta)=$ $\lambda_{j-1}^{*}\left(\phi_{j-1}\right)$ for $j=2, \ldots, K$. Therefore, $\lambda^{*}$ is continuous and strictly mono-decreasing over the entire domain of $\eta$. 